$\begin{array}{ccc} & 2 \mathrm{~A} & 1 \mathrm{KLC} \\ & \mathrm{Z} 5 & \\ \mathrm{c} & + & - \\ \mathrm{d} & + & + \\ \mathrm{f} & + & + \\ \mathrm{g} & + & + \\ \mathrm{h} & + & - \\ \mathrm{i} & + & + \\ \mathrm{j} & + & - \\ \mathrm{k} & + & + \\ \mathrm{l} & - & +\end{array}$

Table 1 Interaction of Picroside $\mathbb{I}$ derivatives and targets

Conclusion In this study, we use Picroside II as leading compound, carried out alkylation, acetylation and amino acid linkage, and 12 derivatives were obtained. And the method of virtual screening was used to screening the hepatoprotective effect of the derivatives. Nine potentially reactive compounds were screened out of twelve derivatives. Compared with traditional cell or animal experiments, this method is simple and fast, saving a lot of time and material resources. Besides, the molecular docking method determine the way of interaction of the target and the compound, and its mechanism was initial explored. This study provides a reference for the further study of the pharmacokinetics and mechanism of action.

Reference

[1].F. Duval, J.E. Morenocuevas, M.T. Gonzálezgarza, C. Rodríguezmontalvo and D.E. Cruzvega Advances in Pharmacological Sciences 2014, 2014 (2014)

[2].J.P. Iredale Journal of Clinical Investigation 117, 3 (2007)

[3].A. Galli, E. Ceni, R. Salzano, T. Mello, D.W. Crabb, G.S. Baroni, F. Ridolfi, C. Surrenti and A. Casini Digestive \& Liver Disease 33, 01 (2001)

[4].E. Ueberham, R. Löw, U. Ueberham, K. Schönig, H. Bujard and R. Gebhardt Hepatology 37, 5 (2003)

[5].Y.M. Subeq, C.Y. Ke, N.T. Lin, C.J. Lee, Y.H. Chiu and B.G. Hsu Cytokine 53, 2 (2011)

\title{
DOI 10.22448/AMJ.2017.3.40-41 \\ EXPOSURE TO HIGH TEMPERATURE CONDITION ENHANCES THE ANTIPYRETIC, ANALGESIC AND ANTI-INFLAMMA- TORY OF SAPOSHNIKOVIA. DIVARICATA FRESH ROOT
}

\author{
Hua Jiang1,2, Jing-Ming Yang2, Ling Cao1,Xiang-Cai Meng1* \\ 1Heilongjiang University of Chinese Medicine, China \\ 2Jinzhou Medical University, Jinzhou 121001, China \\ *Corresponding authors: Prof. Meng Xiang-cai, Ph.D,
}

College of Pharmacy, Heilongjiang University of Chinese Medicine, Heping Road 24, Harbin 150040, China. E-mail: mengxiangcai000@163.com.

\begin{abstract}
It is an ecological environment that leads to the quality difference of Radix saposhnikoviae (RS) produced in different regions or environmental stress. Previous study revealed that exposure of S. divaricata fresh roots to high temperature has potential to increase the chromones content, but it is untrustwrothy to evaluate the quality only depending on components due to complexity. The pharmacokinetics parameters showed that only cimifugin was found in plasma after RS were administered to rats, heat-stress-RS (HRS) had a 50.6\% increase of cimifugin concentration. The anti-pyretic effect of HRS was higher than that of RS at all the dosages, with HRS at $1 \mathrm{~g} / \mathrm{kg}$ nearly equivalent to $2 \mathrm{~g} / \mathrm{kg}$ RS. The analgesic effect of HRS was stronger than that of RS at all the dosages, with HRS at $1 \mathrm{~g} / \mathrm{kg}$ nearly equivalent to $4 \mathrm{~g} / \mathrm{kg}$ RS.The anti-inflammatory effects of the HRS and RS were both dose dependent. The anti-inflammatory effect of HRS was more potent than that of RS at all the dosages, with HRS at $1 \mathrm{~g} / \mathrm{kg}$ nearly equivalent to $2 \mathrm{~g} / \mathrm{kg}$ RS. High temperature dramatically advances the quality of RS.
\end{abstract}

Keywords: Radix Saposhnikoviae; chromone; pharmacokinetics; anti-pyretic; analgesic; anti-inflammatory.

Radix saposhnikoviae (RS), widely used in Asian countries, is the dried root of non-bolting stage of Saposhnikovia divaricata (Turcz.) Schischk and mainly contains diverse chromone components, including cimifugin, prime-O-glucosylcimifugin (PGCN), and 4'-O- $\beta$-D-glucosyl-5-O-methylvisamminol (GML), but only cimifugin can be found in plasma after RS were administered to rats[1]. RS has anti-pyretic, analgesic and anti-inflammatory effects[2], Chromone contents varies tremendously according to the geographical origin or environmental stress, with PGCN varying from $0.11 \%$ to $0.48 \%$, GML from $0.13 \%$ to $0.39 \%$, and the total chromones from $0.18 \%$ to $6.8 \%[3]$. Compared with cultivated RS, higher content of PGCN and GML was found in the wild one. Now, RS is nearly all obtained from the cultivated S. divaricata and the degradation of the quality is an undeniable fact. How to increase the content of the secondary metabolites in RS has become the focus of medicinal herb production.

Herbal medicine quality varied considerably according to the geographical origin or environmental stress, the good cultivated environment habitats can easily lead to reducing quality. Reactive oxygen species are the initial products produced under environmental stress conditions and represent key plant products that induce secondary metabolism, and secondary metabolites are often medicinal ingredients. High temperature must result in production 40 Амурский медицинский журнал №3 (19) 2017 
of excessive reactive oxygen species for the plant. Previous study revealed that exposure of S. divaricata fresh roots to high temperature has potential to increase the chromones content, but RS contain many chromones, each with different activities, it is inappropriate to elevating effect of RS depending on anyone chromone or total chromones.

\section{Objective}

The aim of present study was to confirm the quality-improving effect of high-temperature exposure on RS via examining its pharmacokinetic parameters and in vivo antipyretic, analgesic and anti-inflammatory effects.

\section{Materials and Methods}

The root of RS was placed at $30^{\circ} \mathrm{C}$ for 6 days. The contents of the active constituent were analysed using pharmacokinetics parameters of the chromone derivatives, as measured by high performance liquid chromatography separation and analysis. The antipyretic, analgesic and anti-inflammatory effects were evaluated by pyretic animal model, hot plate test, and ear edema model, respectively.

\section{Results and discussion}

Only cimifugin was found in plasma after RS and heat-stress-RS (HRS) were administered to rats, The cimifugin concentration in these two groups had the same trends, both peaked twice at $1.5 \mathrm{~h}$ and $8 \mathrm{~h}$. Compared with control RS, HRS had a $50.6 \%$ increase of cimifugin concentration (AUCO-24h, $9.41 \mathrm{vs} 6.25 \mu \mathrm{g} /(\mathrm{ml} \cdot \mathrm{h})$. Likewise the anti-pyretic effect of HRS was stronger than that of RS at all the dosages, with HRS at $1 \mathrm{~g} / \mathrm{kg}$ nearly equivalent to $2 \mathrm{~g} / \mathrm{kg} \mathrm{RS}$. A dose dependent increase of the analgesic effect was found with both RS and HRS. The analgesic effect of HRS was stronger than that of RS at all the dosages, with HRS at $1 \mathrm{~g} / \mathrm{kg}$ nearly equivalent to $4 \mathrm{~g} / \mathrm{kg} \mathrm{RS}$. The anti-inflammatory effects of the HRS and RS were both dose dependent. The anti-inflammatory effect of HRS was more potent than that of RS at all the dosages, with HRS at $1 \mathrm{~g} / \mathrm{kg}$ nearly equivalent to $2 \mathrm{~g} / \mathrm{kg} \mathrm{RS}$. The antipyretic, analgesic and anti-inflammatory effect of HRS was overall in agreement with their pharmacokinetics characteristics, the exposure of $\mathrm{S}$. divaricata fresh roots to high temperature can greatly enhance the quality of RS.

\section{References:}

1. Dai JN., Chen XH., Cheng WM. A sensitive liquid chromatography-mass spectrometry method for simultaneous determination of two active chromones from Saposhnikovia root in rat plasma and urine // Joural of Cromatography B. - 2008. -C. 13-19.

2. Chun J.M., Kim H.S., Lee A.Y., Kim S.H., Kim H.K. Anti-Inflammatory and Antiosteoarthritis Effects of Saposhnikovia divaricata ethanol Extract: In Vitro and In Vivo Studies // Evid Based Complement Alternat Med. - 2016. $-\mathrm{C} .1984238$.

3. Zeng LJ., Sun Q.S., Jia L.Y. Content determination of four components from different habitats and different parts of Saposhnikovia divaricata (Turca.) Schischk by PR-HLC // Journal of Shenyang Pharmaceutical University. - 2009. $-\mathrm{C} .127-130$.

\section{DOI 10.22448/AMJ.2017.3.41-42 \\ CLINICAL OBSERVATION OF ACUPUNCTURE COMBINED WITH LASER IN THE TREATMENT OF ALLERGIC RHINITIS HEILONGJIANG UNIVERSITY SECOND AFFILIATED HOSPITAL OF TRADITIONAL CHINESE MEDICINE}

\section{Jiang Shanshan}

Abstract Allergic rhinitis (allergic rhinitis), also known as allergic rhinitis, allergic rhinitis, is a kind of inhalation of external allergens caused by nasal mucosa to type I allergy-based non-infectious inflammation, clinical nose Itching, shouting, runny nose as the main symptoms. According to the different onset time, can be divided into seasonal allergic rhinitis (seasonal allergic rhinitis) and perennial allergic rhinitis (perennial allergic rhinitis) two categories. The former often caused by seasonal sensitizers, such as pollen, pollen allergic rhinitis only in the spread of pollen season, it is also known as pollen disease (pollinosis); the latter often caused by perennial sensitizers, such as the house Dust, debris, animal dander, fungi and so on. At present, the prevalence of our country is about $10 \%$. In recent years, China's rapid economic development, the prevalence of allergic rhinitis also showed a significant increase in the trend, although it is not a fatal disease, but can seriously affect the quality of life of patients, to the daily life and work caused a greater negative impact The At present, Western medicine treatment of allergic rhinitis with immunotherapy and drug therapy, drug treatment to antihistamines, immunosuppressive agents, leukotriene receptor antagonist, anticholinergic drugs, glucocorticoids, etc., although the symptoms improved faster, However, toxic side effects, such as gastrointestinal stimulation, immune function inhibition and hormone side effects, and these drugs after the illness often repeated, difficult to cure, resulting in poor compliance. In recent years, Chinese and foreign medicine treatment of the disease reported in the literature more, and many treatment methods, the effect is more significant, especially acupuncture treatment of the disease has a positive effect, good results and stable, the advantages of small side effects, combined with laser therapy makes clinical efficacy More significant, won the general recognition, showing the advantages of traditional Chinese medicine treatment of allergic rhinitis.

Key words: acupuncture combined with laser,allergic rhinitis,clinical Observation

Objective This project chooses the research method which is compatible with the research content, and selects 